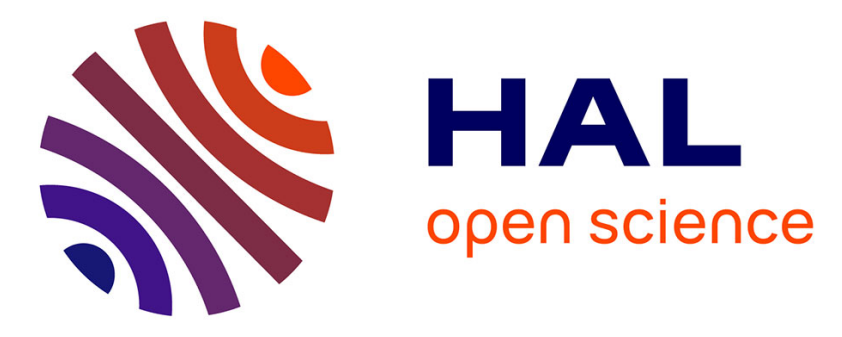

\title{
A new generalized power flow method for multi connected DC grids
}

\author{
Eduardo Jimenez, Miguel Jimenez-Carrizosa Jimenez Carrizosa, Abdelkrim \\ Benchaib, Gilney Damm, Françoise Lamnabhi-Lagarrigue
}

\section{To cite this version:}

Eduardo Jimenez, Miguel Jimenez-Carrizosa Jimenez Carrizosa, Abdelkrim Benchaib, Gilney Damm, Françoise Lamnabhi-Lagarrigue. A new generalized power flow method for multi connected DC grids. International Journal of Electrical Power \& Energy Systems, 2016, 74, pp.329-337. 10.1016/j.ijepes.2015.07.032 . hal-01260078

\section{HAL Id: hal-01260078 \\ https://hal-centralesupelec.archives-ouvertes.fr/hal-01260078}

Submitted on 21 Sep 2020

HAL is a multi-disciplinary open access archive for the deposit and dissemination of scientific research documents, whether they are published or not. The documents may come from teaching and research institutions in France or abroad, or from public or private research centers.
L'archive ouverte pluridisciplinaire HAL, est destinée au dépôt et à la diffusion de documents scientifiques de niveau recherche, publiés ou non, émanant des établissements d'enseignement et de recherche français ou étrangers, des laboratoires publics ou privés. 


\title{
A new generalized power flow for multi connected DC grids.
}

\author{
E. Jiménez ${ }^{1}$, M. Jiménez Carrizosa ${ }^{2}$, A. Benchaib ${ }^{3}$, G. Damm ${ }^{4}$ and F. Lamnabhi-Lagarrigue ${ }^{2}$ \\ ${ }^{1}$ Universidad de Castilla- La Mancha, UCLM, Albacete, Spain. \\ email: Eduardo.Jimenez@uclm.es \\ ${ }^{2}$ Laboratoire des signaux et systèmes, SUPELEC, France. \\ email: Miguel.Jimenez@lss.supelec.fr, lamnabhi@lss.supelec.fr \\ ${ }^{3}$ ALSTOM GRID / Cnam, France. \\ email: abdelkrim.benchaib@alstom.com \\ ${ }^{4}$ Laboratoire Informatique, Biologie Intégrative et Systèmes Complex, IBISC, France. \\ email: gilney.damm@ibisc.fr
}

\section{Acknowledgments}

This work is supported by WINPOWER project (ANR-10-SEGI-016).

\begin{abstract}
A new power flow for DC grids is presented in this paper. With this new methodology to have more than one node where the voltages are known is possible, in contrast to old methods where there was a node where the voltage was specified (the slack bus). A complete proof is given, which guarantees the unique existence of solutions. This new algorithm could be easily adapted for AC systems with the explained philosophy in this paper. Some simulations are tested in order to show the power of this new tool. In addition, a detailed study of the variation of the power and voltages when these variables change is shown.
\end{abstract}

\section{Keywords}

Power flow, DC grids, nonlinear systems, contraction mapping theorem.

\section{Introduccion}

In electrical networks with real load and generation, the use of the power flow is crucial for the proper functioning of the system. The goal of a power 
flow study, basically, is to obtain complete voltage and power informations for each bus in the grid in steady state. However it may perform other types of analysis, such as short-circuit fault analysis, stability studies, unit commitment or economic dispatch.

Energy transmission has historically been carried out in alternative current (AC). However high voltage direct current (HVDC) transmission is investigated in this study due to several advantages it possesses over $\mathrm{AC}$ lines.First of all, reactive power makes AC transmission losses greater in offshore locations than on land. Additionally, transmission capacity is also greater in HVDC lines due to the non-existence of the skin effect, and also the efficiency and controllability of DC converters are higher [1-4]. Another advantage of using HVDC is that the use of fewer cables in DC lines also implies lower costs and weight enabling therefore the possibility of operating in remote marine regions where wind conditions are even more favorable [5,6].

In $\mathrm{AC}$ power systems, power flow problem is defined by nonlinear and non-convex equations. In HVDC systems where there is no reactive power involved, the power flow problem is less complex but still retains its nonlinear characteristic when voltages control are included in the formulation.

There are several different methods of solving the resulting nonlinear system of equations. The most popular is the well known Newton-Raphson method [7]. This method presents an important property: the solutions can be easily obtained trough the equations' linearization. An important disadvantage is that the convergence of the method is not always guaranteed. Furthermore, in the case of power systems it is necessary to consider a slack bus to apply this method. This fact entails risks for the proper operation of the system, such as the loss of the slack bus (for example a communication lost), that would cause the loss of the reference and consequently the abandon of the equilibrium because the method is not applicable. With this new algorithm this risk disappears, because more than one node could be voltage reference.

In 8 an optimal power flow problem for HVDC systems with predictive control tools is shown. It uses a geometrical proof for the case of a system with 3 nodes, but it lacks a strict mathematical proof for the case of $n$ nodes, which is shown in our paper.

This paper is outlined as follows: in section $\mathbf{I}$ an introduction and the background information of the study is presented. Section II presents some definitions and basic relations which will help us to define better our problem. In section III the two main properties will be shown, as well as their respective proofs. In section IV some simulations are shown. In section section $\mathbf{V}$ the conclusions are explained. Finally in annex some mathematical relations uses in this work are shown. 


\section{Definitions and basic relations.}

We have considered a passive network with $n$ nodes $(n \geq 2)$. This grid is connected because any two nodes of the network are connected by at least one path formed by branches of the network. The lines are bipolar since they have two phases (+ and -) as shows figure 1.

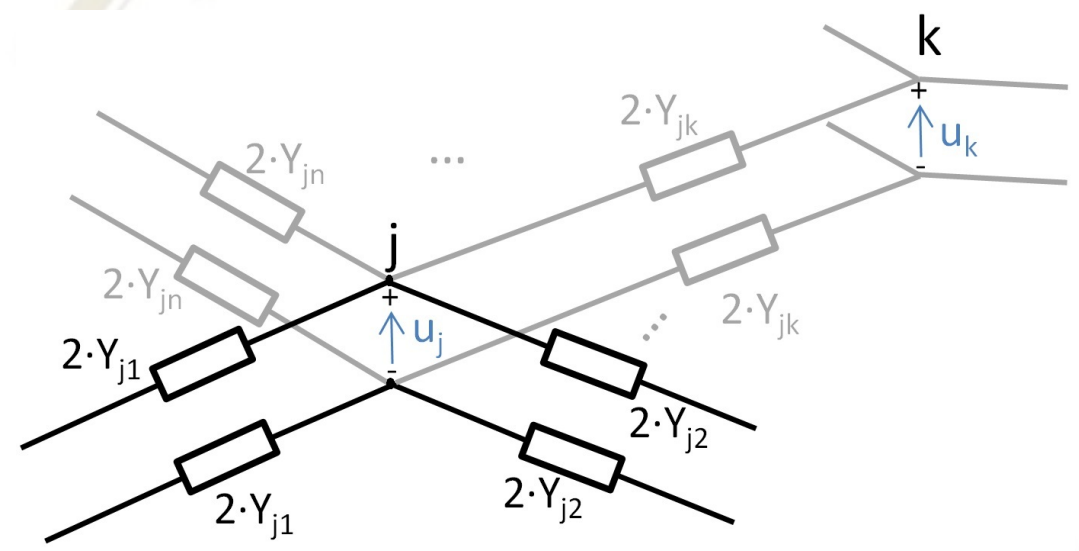

Fig. 1: Figure

Definition 1. $\forall j, k \in\{1,2, \ldots, n\}, j \neq k, y_{j, k}$ is the admittance of the branch which connects node $j$ with node $k$. It corresponds with the two conductors (positive and negative). When the branch exits then $y_{j, k}>0$, whereas if there is no branch $y_{j, k}=0$. It holds that $y_{j, k}=y_{k, j}$.

Definition 2. $\forall j \in\{1,2, \ldots, n\}, y_{j, j}$ is the sum of the admittances of the network which converge at node $j$.

$$
y_{j, j}=\sum_{k=1(k \neq j)}^{n} y_{j, k}
$$

Definition 3. $u_{j}$ is the voltage between positive and negative terminals of node $j$.

Definition 4. $i_{j}$ is the current which comes in the network through the positive terminal of node $j$. (Its value is negative when the current leaves the grid).

Definition 5. $P_{j}$ is the power which comes in the network at node $j$. (Its value is negative when the power leaves the grid).

Next some basic relationship are explained. The first one is that, in steady state, the system of equations shown in 2 are satisfied:

Winpower/Alstom days - 5 \& 6 Ferbuary 2014. CONFIDENTIAL USE. NOT PUBLISHED 


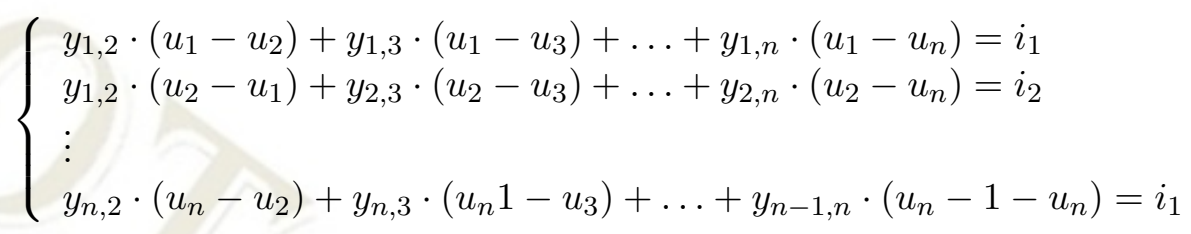

which is equivalent to system shown in (3)

$$
\left\{\begin{array}{r}
y_{1,1} \cdot u_{1}-y_{1,2} \cdot u_{2}-y_{1,3} \cdot u_{3}-\ldots-y_{1, n} \cdot u_{n}=i_{1} \\
-y_{1,2} \cdot u_{1}+y_{2,2} \cdot u_{2}-y_{2,3} \cdot u_{3}-\ldots-y_{2, n} \cdot u_{n}=i_{2} \\
\vdots \\
-y_{1, n} \cdot u_{1}+y_{2, n} \cdot u_{2}-y_{3, n} \cdot u_{3}-\ldots+y_{n, n} \cdot u_{n}=i_{n}
\end{array}\right.
$$

In matrix form:

$$
\mathbf{Y} \cdot \mathbf{u}=\mathbf{i}
$$

Moreover:

$$
P_{1}=u_{1} \cdot i_{1}, P_{2}=u_{2} \cdot i_{2}, \ldots, P_{n}=u_{n} \cdot i_{n}
$$

and consequently

$$
\left\{\begin{array}{r}
y_{1,1} \cdot u_{1}^{2}-y_{1,2} \cdot u_{1} \cdot u_{2}-y_{1,3} \cdot u_{1} \cdot u_{3}-\ldots-y_{1, n} \cdot u_{1} \cdot u_{n}=P_{1} \\
-y_{1,2} \cdot u_{1} \cdot u_{2}+y_{2,2} \cdot u_{2}^{2}-y_{2,3} \cdot u_{2} \cdot u_{3}-\ldots-y_{2, n} \cdot u_{2} \cdot u_{n}=P_{2} \\
\vdots \\
-y_{1, n} \cdot u_{1} \cdot u_{n}+y_{2, n} \cdot u_{2} \cdot u_{n}-y_{3, n} \cdot u_{3} \cdot u_{n}-\ldots+y_{n, n} \cdot u_{n}^{2}=P_{n}
\end{array}\right.
$$

Moreover, it is also true that:

$$
\mathbf{u}^{t} \cdot \mathbf{Q}_{j} \mathbf{u}=\mathbf{P}_{j} \quad \forall j \in\{1,2, \ldots, n\}
$$

and consequently,

$$
\mathbf{u}^{t} \cdot \mathbf{Y u}=\sum_{j=1}^{n} \mathbf{P}_{j}
$$

where $\mathbf{i}=\left[i_{1}, i_{2}, \ldots, i_{n}\right]^{t}, \mathbf{u}=\left[u_{1}, u_{2}, \ldots, u_{n}\right]^{t}, \mathbf{P}=\left[P_{1}, P_{2}, \ldots, P_{n}\right]^{t}, \mathbf{Y}$ is the admittance matrix: 


$$
\mathbf{Y}=\left[\begin{array}{cccc}
y_{1,1} & -y_{1,2} & \ldots & -y_{1, n} \\
-y_{1,2} & y_{2,2} & \ldots & -y_{2, n} \\
\vdots & \vdots & \ddots & \vdots \\
-y_{1, n} & -y_{2, n} & \cdots & y_{n, n}
\end{array}\right]
$$

and $\mathbf{Q}_{j}$ has the form:

$$
\mathbf{Q}_{j}=\left[\begin{array}{ccccccc}
0 & \ldots & 0 & -\frac{y_{1, j}}{2} & 0 & \ldots & 0 \\
\vdots & \ddots & \vdots & \vdots & \ldots & \vdots & \vdots \\
0 & \ldots & 0 & -\frac{y_{j-1, j}}{2} & 0 & \ldots & 0 \\
-\frac{y_{1, j}}{2} & \ldots & -\frac{y_{j-1, j}}{2} & y_{j, j} & -\frac{y_{j, j+1}}{2} & \vdots & -\frac{y_{j, n}}{2} \\
0 & \ldots & 0 & -\frac{y_{j, j+1}}{2} & 0 & \ldots & 0 \\
\vdots & \vdots & \vdots & \vdots & \ldots & \ddots & \vdots \\
0 & \ldots & 0 & -\frac{y_{j, n}}{2} & 0 & \ldots & 0
\end{array}\right]
$$

\subsection{Basic properties}

Next some basic properties are explained and detailed.

Basic property 1. If we know the voltages on all nodes, we can find all currents and powers.

Basic property 2. As $i_{1}+i_{2}+\ldots+i_{n}=0$, then if we know the entering currents in $n-1$ nodes, we can find the entering current in the remaining node.

Basic property 3. $P_{1}+P_{2}+\ldots+P_{n} \geq 0\left(P_{1}+P_{2}+\ldots+P_{n}\right.$ is the lost power in the network)

If $u_{1}=u_{2}=\ldots=u_{n}$, then $i_{1}=\ldots=i_{n}=0$ and $P_{1}+P_{2}+\ldots+P_{n}=0$.

If $\exists j, k \in\{1,2, \ldots, n\}$ such that $u_{j} \neq u_{k}$ then $P_{1}+P_{2}+\ldots+P_{n} \geq 0$.

Basic property 4. The matrix $\boldsymbol{Y}$ is positive semidefinite of rank $n-1$.

$\forall j \in\{1,2, \ldots, n\}$ the matrix of order $n-1$ that results to remove row $j$ and column $j$ of $\boldsymbol{Y}$ is positive definite.

$\forall j \in\{1,2, \ldots, n-1\}$ matrix $\boldsymbol{Y}_{k}$, formed by the elements of the first $k$ rows and first $k$ columns of $\boldsymbol{Y}$, and matrix $\boldsymbol{\Lambda}_{\mathbf{n}-\mathbf{k}}$ formed by the elements of the last $n-k$ rows and last $n-k$ columns of $\boldsymbol{Y}$, are definite positive.

$$
\boldsymbol{Y}_{k}=\left[\begin{array}{ccc}
y_{1,1} & \ldots & -y_{1, n} \\
-y_{1,2} & \ldots & -y_{2, n} \\
\vdots & \ddots & \vdots \\
-y_{1, n} & \cdots & y_{n, n}
\end{array}\right] \quad \boldsymbol{\Lambda}_{\mathbf{n}-\mathbf{k}}=\left[\begin{array}{ccc}
y_{k+1, k+1} & \ldots & -y_{k+1, n} \\
-y_{k+1,2} & \ldots & -y_{k+2, n} \\
\vdots & \ddots & \vdots \\
-y_{k+1, n} & \ldots & y_{n, n}
\end{array}\right]
$$

Winpower/Alstom days -5 \& 6 Ferbuary 2014. CONFIDENTIAL USE. NOT PUBLISHED 
Basic property 5. $\forall j \in\{1,2, \ldots, n\}$ matrix $\boldsymbol{Q}_{j}$ is indefinite of order 2. That means, $\boldsymbol{Q}_{j}$ has a positive eigenvalue, other negative, and the remaining $n-2$ are null.

Basic property 6. If we know the entering currents in $k$ nodes, with $0<$ $k<n$, and the voltages in the other $n-k$ nodes, the voltages in all nodes are uniquely determined, and hence also the currents and powers. Since, known $i_{1}, \ldots, i_{k}, u_{k+1}, \ldots, u_{n}$, values of $u_{1}, \ldots, u_{k}$ are solutions of linear system shown in (12) whose coefficient matrix $\boldsymbol{Y}_{k}$ is invertible.

$$
\left\{\begin{array}{r}
y_{1,1} \cdot u_{1}-y_{1,2} \cdot u_{2}-\ldots-y_{1, k} \cdot u_{k}=i_{1}+y_{1, k+1} \cdot u_{k+1}+\ldots+y_{1, n} \cdot u_{n} \\
-y_{1,2} \cdot u_{1}+y_{2,2} \cdot u_{2}-\ldots-y_{2, k} \cdot u_{k}=i_{2}+y_{2, k+1} \cdot u_{k+1}+\ldots+y_{2, n} \cdot u_{n} \\
\vdots \\
-y_{1, k} \cdot u_{1}-y_{2, k} \cdot u_{2}-\ldots+y_{k, k} \cdot u_{k}=i_{k}+y_{k, k+1} \cdot u_{k+1}+\ldots+y_{k, n} \cdot u_{n}
\end{array}\right.
$$

Basic property 7. If we know the entering powers in $k$ nodes, with $\quad 0<$ $k<n$, and the voltages in the other $n-k$ nodes, for sufficiently high values of voltages, we can find the remaining voltages, and therefore also the currents and powers in all nodes.

Effectively, if the known voltage values $u_{k+1}, \ldots, u_{n}$ are close to nominal voltage value $U_{n}$ of the network, and this is sufficiently high, the unknown voltage values $u_{1}, \ldots, u_{k}$ are also close to $U_{n}$, and they will be unique. In effect, if we know $P_{1}, \ldots, P_{k}, u_{k+1}, \ldots, u_{n}$, the values of $u_{1}, \ldots, u_{k}$ are the solution of the following system:

$$
\left\{\begin{array}{r}
y_{1,1} \cdot u_{1}-y_{1,2} \cdot u_{2}-\ldots-y_{1, k} \cdot u_{k}=\frac{P_{1}}{u_{1}}+y_{1, k+1} \cdot u_{k+1}+\ldots+y_{1, n} \cdot u_{n} \\
-y_{1,2} \cdot u_{1}+y_{2,2} \cdot u_{2}-\ldots-y_{2, k} \cdot u_{k}=\frac{P_{2}}{u_{2}}+y_{2, k+1} \cdot u_{k+1}+\ldots+y_{2, n} \cdot u_{n} \\
\vdots \\
-y_{1, k} \cdot u_{1}-y_{2, k} \cdot u_{2}-\ldots+y_{k, k} \cdot u_{k}=\frac{P_{k}}{u_{k}}+y_{k, k+1} \cdot u_{k+1}+\ldots+y_{k, n} \cdot u_{n}
\end{array}\right.
$$

and applying the following Property 1 the mentioned results in this basic property 7 are obtained.

\section{Main properties}

In this section the two main results of this paper are formulated and proven.

\section{Property 1.}

- Let $k \in \mathbb{N}$ be such that $0<k<n$. 
- Let $\boldsymbol{Y}_{k} \in \mathbb{R}^{k \times k}$ be such that:

$$
\boldsymbol{Y}_{k}=\left[\begin{array}{ccc}
y_{1,1} & \ldots & -y_{1, k} \\
-y_{1,2} & \ldots & -y_{2, k} \\
\vdots & \ddots & \vdots \\
-y_{1, k} & \ldots & y_{k, k}
\end{array}\right]
$$

- Let $\boldsymbol{\Gamma}_{\mathbf{k}} \in \mathbb{R}^{k \times(n-k)}$ be such that:

$$
\boldsymbol{\Gamma}_{\mathbf{k}}=\left[\begin{array}{cccc}
y_{1, k+1} & y_{1, k+2} & \ldots & y_{1, n} \\
y_{2, k+1} & y_{2, k+2} & \ldots & y_{2, n} \\
\vdots & \vdots & \ddots & \vdots \\
y_{k, k+1} & y_{k, k+2} & \ldots & y_{k, n}
\end{array}\right]
$$

- Let $\boldsymbol{\Lambda}_{\mathbf{n}-\mathbf{k}} \in \mathbb{R}^{(n-k) \times(n-k)}$ be such that:

$$
\boldsymbol{\Lambda}_{\mathbf{n}-\mathbf{k}}=\left[\begin{array}{ccc}
y_{k+1, k+1} & \ldots & -y_{k+1, n} \\
-y_{k+1,2} & \ldots & -y_{k+2, n} \\
\vdots & \ddots & \vdots \\
-y_{k+1, n} & \cdots & y_{n, n}
\end{array}\right]
$$

- Let $\boldsymbol{Y} \in \mathbb{R}^{n \times n}$ be such that:

$$
\boldsymbol{Y}=\left[\begin{array}{cc}
\boldsymbol{Y}_{k} & -\boldsymbol{\Gamma}_{\mathbf{k}} \\
-\boldsymbol{\Gamma}_{\mathrm{k}}^{\mathrm{t}} & \boldsymbol{\Lambda}_{\mathbf{n}-\mathbf{k}}
\end{array}\right]
$$

- Let $P_{1} \ldots, P_{k}$ be the entering power in the first $k$ nodes, and let $P$ be $P=\max \left\{\left|P_{j}\right| \quad / \quad 1 \leq j \leq k\right\}$.

- Let $c, \epsilon, \delta \in \mathbb{R}$ be such that, $0<c<1,0<\epsilon$ and $0<\delta \leq \frac{\epsilon}{2\left\|\boldsymbol{Y}_{k} \cdot \boldsymbol{\Gamma}_{\mathbf{k}}\right\|_{\infty}}$.

- Let $u_{0}, u_{N} \in \mathbb{R}$ be such that $u_{0} \geq \max \left\{\frac{2 \cdot P \cdot\left\|\boldsymbol{Y}_{k}^{-1}\right\|_{\infty}}{\epsilon}, \sqrt{\frac{P \cdot\left\|\boldsymbol{Y}_{k}^{-1}\right\|_{\infty}}{c}}\right\}$, with $u_{0}>0$ and $u_{N}>u_{0}+\epsilon>u_{0}$.

- $D=\left\{\left(u_{1}, \ldots, u_{k}\right)^{t} \in \mathbb{R}^{k}: u_{1} \geq u_{0}, \ldots, u_{k} \geq u_{0}\right\}$

- Let $\Psi: D \rightarrow \mathbb{R}^{k}$ be the function defined by $\Psi\left(\left(u_{1}, \ldots, u_{k}\right)^{t}\right)=\left(\frac{P_{1}}{u_{1}}, \ldots, \frac{P_{k}}{u_{k}}\right)^{t}$

- Let $V_{N}, W_{N}$ be such that

$$
V_{N}=\left[\begin{array}{c}
u_{N} \\
\vdots \\
u_{N}
\end{array}\right] \in \mathbb{R}^{k}, \quad W_{N}=\left[\begin{array}{c}
u_{N} \\
\vdots \\
u_{N}
\end{array}\right] \in \mathbb{R}^{n-k}
$$


With these conditions is true that for any $W=\left(w_{k+1}, \ldots, w_{n}\right)^{t} \in \bar{B}_{\infty}\left(W_{N}, \delta\right){ }^{1}$ there exists a unique $V=\left(v_{1}, \ldots, v_{k}\right)^{t} \in D$ such that hold:

1.- $\left\{\begin{array}{r}y_{1,1} \cdot v_{1}-y_{1,2} \cdot v_{2}-\ldots-y_{1, k} \cdot v_{k}=\frac{P_{1}}{v_{1}}+y_{1, k+1} \cdot w_{k+1}+\ldots+y_{1, n} \cdot w_{n} \\ -y_{1,2} \cdot v_{1}+y_{2,2} \cdot v_{2}-\ldots-y_{2, k} \cdot v_{k}=\frac{P_{2}}{v_{2}}+y_{2, k+1} \cdot w_{k+1}+\ldots+y_{2, n} \cdot w_{n} \\ \vdots \\ -y_{1, k} \cdot v_{1}-y_{2, k} \cdot v_{2}-\ldots+y_{k, k} \cdot v_{k}=\frac{P_{k}}{v_{k}}+y_{k, k+1} \cdot w_{k+1}+\ldots+y_{k, n} \cdot w_{n}\end{array}\right.$

2.- $V \in \bar{B}_{\infty}\left(W_{N}, \epsilon\right)$

3.- If $\left(s_{j}\right)_{j \in \mathbb{N}}$ is a succession defined by: $s_{j+1}=Y_{k}^{-1} \cdot \Psi\left(s_{j}\right)+Y_{k}^{-1} \cdot \Gamma_{k} \cdot W$, where $s_{0} \in D$ and $\forall j \in \mathbb{N}$, it holds:

$$
\begin{aligned}
& \text { 3.1.- } V=\lim _{j \rightarrow 0} s_{j} \\
& \text { 3.2.- }\left\|s_{j}-V\right\|_{\infty} \leq\left\|s_{2}-s_{1}\right\|_{\infty} \cdot \frac{c^{j-1}}{1-c} \leq 2 \cdot \epsilon \cdot \frac{c^{j-1}}{1-c} \quad \forall j \in \mathbb{N}^{*}
\end{aligned}
$$

\section{Proof}

1.- If we term $v=\left(u_{1}, \ldots, u_{k}\right)^{t} \in D$, and let $g$ be the mapping such that $g: D \rightarrow D, g(v)=\mathbf{Y}_{k}^{-1} \cdot \Psi(v)+\mathbf{Y}_{k}^{-1} \cdot \Gamma_{k} \cdot W \forall v \in D$, then when we know the voltages $u_{k+1}=w_{k+1}, \ldots, u_{n}=w_{n}$ the system shown in (19) is equivalent to:

$$
\mathbf{Y}_{k} \cdot v=\Psi(v)+\Gamma_{k} \cdot W
$$

which is equivalent to

$$
v=\mathbf{Y}_{k}^{-1} \cdot \Psi(v)+\mathbf{Y}_{k}^{-1} \cdot \Gamma_{k} \cdot W
$$

and this is equivalent to

$$
v=g(v)
$$

so $v$ is a solution of $(20)$ if and only if it is a fixed point of the mapping $g$.

Let us check that $g$ is a contractive application in D. First we check that $\forall v \in D, g(v) \in D$, because

$$
g(v)=\mathbf{Y}_{k}^{-1} \cdot \Psi(v)+\mathbf{Y}_{k}^{-1} \cdot \Gamma_{k} \cdot W
$$

from (2) and (3) is easy to realize that it fulfills:

$$
\mathbf{Y}_{k} \cdot V_{N}=\Gamma_{k} \cdot W_{N}
$$

and consequently:

$$
V_{N}=\mathbf{Y}_{k}^{-1} \cdot \Gamma_{k} \cdot W_{N}
$$

\footnotetext{
${ }^{1}$ See annex.

Winpower/Alstom days - 5 \& 6 Ferbuary 2014. CONFIDENTIAL USE. NOT PUBLISHED
} 
from $(23)$ and 25 we obtain:

$$
g(v)-V_{N}=\mathbf{Y}_{k}^{-1} \cdot \Psi(v)+\mathbf{Y}_{k}^{-1} \cdot \Gamma_{k} \cdot\left(W-W_{N}\right)
$$

and consequently:

$$
\left\|g(v)-V_{N}\right\|_{\infty} \leq\left\|\mathbf{Y}_{k}^{-1}\right\|_{\infty} \cdot\|\Psi(v)\|_{\infty}+\left\|\mathbf{Y}_{k}^{-1} \cdot \Gamma_{k}\right\|_{\infty} \cdot\left\|W-W_{N}\right\|_{\infty}
$$

from (27), and taking into account that:

$$
\|\Psi(v)\|_{\infty}=\max \left\{\frac{\left|P_{j}\right|}{u_{j}}: 1 \leq j \leq k\right\} \leq \frac{P}{u_{0}} \leq \frac{\epsilon}{2 \cdot\left\|\mathbf{Y}_{k}^{-1}\right\|_{\infty}}
$$

and also that:

$$
\left\|W-W_{N}\right\|_{\infty} \leq \delta \leq \frac{\epsilon}{2 \cdot\left\|\mathbf{Y}_{k}^{-1} \cdot \Gamma_{k}\right\|_{\infty}}
$$

we may deduce that

$$
\left\|g(v)-V_{N}\right\|_{\infty} \leq \delta \leq \frac{\epsilon}{2}+\frac{\epsilon}{2}=\epsilon
$$

which is equivalent to:

$$
g(v) \in \bar{B}_{\infty}\left(V_{N}, \epsilon\right)
$$

and taking into account that:

$$
\bar{B}_{\infty}\left(V_{N}, \epsilon\right) \subset D
$$

it is true that:

$$
g(v) \in D
$$

Secondly, $\forall x, y \in D$ it is clear that $\|g(x)-g(y)\|_{\infty} \leq c \cdot\|x-y\|_{\infty}$, due to:

$$
\begin{aligned}
& g(x)=\mathbf{Y}_{k}^{-1} \cdot \Psi(x)+\mathbf{Y}_{k}^{-1} \cdot \Gamma_{k} \cdot W \\
& g(y)=\mathbf{Y}_{k}^{-1} \cdot \Psi(y)+\mathbf{Y}_{k}^{-1} \cdot \Gamma_{k} \cdot W
\end{aligned}
$$

and therefore:

$$
\|g(x)-g(y)\|_{\infty}=\left\|\mathbf{Y}_{k}^{-1} \cdot(\Psi(x)-\Psi(y))\right\|_{\infty} \leq\left\|\mathbf{Y}_{k}^{-1}\right\|_{\infty} \cdot\|\Psi(x)-\Psi(y)\|_{\infty}(36)
$$


beside

$$
\Psi(x)-\Psi(y)=\left[\begin{array}{c}
\frac{P_{1} \cdot\left(y_{1}-x_{1}\right)}{x_{1} \cdot y_{1}} \\
\vdots \\
\frac{P_{k} \cdot\left(y_{k}-x_{k}\right)}{x_{k} \cdot y_{k}}
\end{array}\right] \Rightarrow\|\Psi(x)-\Psi(y)\|_{\infty} \leq c \cdot\|x-y\|_{\infty}
$$

and as $0<c<1$ it holds that $g$ is a contractive mapping in $D$.

Taking into account that $D$ is a closed set, the fixed-point theorem of a contractive application ensures that there exists a single point $V=$ $\left(v_{1}, \ldots, v_{k}\right)^{t} \in D$ such that $g(V)=V$, that means, $V$ is fixed point of $g$, and by the above explanation this is the unique solution vector from equation (20) and system (19).

2.- Result 2 of property 1 is satisfied because $V=g(V) \in D$ and $g(v) \in$ $\bar{B}_{\infty}\left(V_{N}, \epsilon\right)$ according to 31$)$.

3.- The fixed-point theorem of contractive application also ensures that if $\left(s_{j}\right)_{j \in \mathbb{N}}$ is a succession defined by: $s_{0} \in D$ and $\forall j \in \mathbb{N}, s_{j+1}=g\left(s_{j}\right)$, the only fixed point $\mathrm{V}$ holds:

a) $\mathrm{V}=\lim _{j \rightarrow 0} s_{j}$

b) $\left\|s_{j}-V\right\|_{\infty} \leq\left\|s_{2}-s_{1}\right\|_{\infty} \cdot \frac{c^{j-1}}{1-c} \leq 2 \cdot \epsilon \cdot \frac{c^{j-1}}{1-c} \quad \forall j \in \mathbb{N}^{*}$

Taking into account also that $s_{1}=g\left(s_{0}\right) \in \bar{B}_{\infty}\left(V_{N}, \epsilon\right)$ and $s_{2}=g\left(s_{1}\right) \in$ $\bar{B}_{\infty}\left(V_{N}, \epsilon\right)$, we verify that:

$$
\left\|s_{2}-s_{1}\right\|_{\infty} \leq 2 \cdot \epsilon
$$

so it is true that:

$$
\left\|s_{j}-V\right\|_{\infty} \leq\left\|s_{2}-s_{1}\right\|_{\infty} \cdot \frac{c^{j-1}}{1-c} \leq 2 \cdot \epsilon \cdot \frac{c^{j-1}}{1-c} \quad \forall j \in \mathbb{N}^{*}
$$

Property 2. If $P_{1}, P_{2}, \ldots, P_{k}$ remain constant in 19 , considering the application that goes from $W$ to $V$ and calling $\Pi=\left(P_{k+1}, \ldots, P_{n}\right)^{t}$,

$$
\frac{\partial V}{\partial W}=\left[\begin{array}{cccc}
\frac{\partial V_{1}}{\partial w_{k+1}} & \frac{\partial V_{1}}{\partial w_{k+2}} & \cdots & \frac{\partial V_{1}}{\partial w_{n}} \\
\frac{\partial V_{2}}{\partial w_{k+1}} & \frac{\partial V_{2}}{\partial w_{k+2}} & \cdots & \frac{\partial V_{2}}{\partial w_{n}} \\
\vdots & \vdots & \ddots & \vdots \\
\frac{\partial V_{k}}{\partial w_{k+1}} & \frac{\partial V_{k}}{\partial w_{k+2}} & \cdots & \frac{\partial V_{k}}{\partial w_{n}}
\end{array}\right] \quad \quad \quad \frac{\partial \Pi}{\partial W}=\left[\begin{array}{ccccc}
\frac{\partial P_{k+1}}{\partial w_{k+1}} & \frac{\partial P_{k+1}}{\partial w_{k+2}} & \cdots & \frac{\partial P_{k+1}}{\partial w_{n}} \\
\frac{\partial P_{k+2}}{\partial w_{k+1}} & \frac{\partial P_{k+2}}{\partial w_{k+2}} & \cdots & \frac{\partial P_{k+2}}{\partial w_{n}} \\
\vdots & \vdots & \ddots & \vdots \\
\frac{\partial P_{n}}{\partial w_{k+1}} & \frac{\partial P_{n}}{\partial w_{k+2}} & \cdots & \frac{\partial P_{n}}{\partial w_{n}}
\end{array}\right]
$$

it is also true that:

- The jacobian matrix $\frac{\partial V}{\partial W}$ holds the following premise:

$$
\left[\boldsymbol{Y}_{k}-\Psi^{\prime}(V)\right] \cdot \frac{\partial V}{\partial W}=\Gamma_{k}
$$


where $\Psi^{\prime}(V)=\frac{\partial \Psi}{\partial V}$ the jacobian matrix of $\Psi$. Besides if $\left[\boldsymbol{Y}_{k}-\Psi^{\prime}(V)\right]$ is invertible then:

$$
\frac{\partial V}{\partial W}=\left[\boldsymbol{Y}_{k}-\Psi^{\prime}(V)\right]^{-1} \cdot \Gamma_{k}
$$

- The jacobian matrix $\frac{\partial \Pi}{\partial W}$ is determined by the following expression:

$$
\frac{\partial \Pi}{\partial W}=[\Phi]+[W] \cdot\left[\Lambda_{n-k}-\Gamma_{k}^{t} \cdot \frac{\partial V}{\partial W}\right]
$$

where $[\Phi]=\operatorname{diag}\left(\frac{P_{k+1}}{w_{k+1}}, \frac{P_{k+2}}{w_{k+2}}, \ldots, \frac{P_{n}}{w_{n}}\right)$ and $[W]=\operatorname{diag}\left(w_{k+1}, w_{k+2}, \ldots, w_{n}\right)$, so if $\left[\boldsymbol{Y}_{k}-\Psi^{\prime}(V)\right]$ is invertible then:

$$
\frac{\partial \Pi}{\partial W}=[\Phi]+[W] \cdot\left[\Lambda_{n-k}-\Gamma_{k}^{t} \cdot\left[\boldsymbol{Y}_{k}-\Psi^{\prime}(V)\right]^{-1} \cdot \Gamma_{k}\right]
$$

\section{Proof}

The system $(19)$ is equivalent to:

$$
\mathbf{Y}_{k} \cdot V=\Psi(V)+\Gamma_{k} \cdot W
$$

and if $\Psi(V)=\frac{\partial \Psi}{\partial V}$ is the jacobian matrix of $\Psi$, it holds that:

$$
\mathbf{Y}_{k} \cdot \frac{\partial \Psi}{\partial V}=\Psi^{\prime}(V) \cdot \frac{\partial \Psi}{\partial V}+\Gamma_{k}
$$

which is equivalent to equation (41), and if in addition $\left[\mathbf{Y}_{k}-\Psi^{\prime}(V)\right]$ is invertible equation 42 is verified.

When $u_{1}=v_{1}, \ldots, u_{k}=v_{k}, u_{k+1}=w_{k+1}, \ldots, u_{n}=w_{n}$, from system (6) we obtain:

$$
\left\{\begin{array}{l}
-y_{1, k+1} \cdot v_{1} \cdot w_{k+1}-\ldots-y_{k, k+1} \cdot v_{k} \cdot w_{k+1}+y_{k+1, k+1} \cdot w_{k+1}^{2}-\ldots-y_{k+1, n} \cdot w_{k+1} \cdot w_{n}=P_{k+1} \\
\vdots \\
-y_{1, n} \cdot v_{1} \cdot w_{n}-\ldots-y_{k, n} \cdot v_{k} \cdot w_{n}-y_{k+1, n} \cdot w_{k+1} \cdot w_{n}-\ldots+y_{n, n} \cdot w_{n}^{2}=P_{n}
\end{array}\right.
$$

ans due to $w_{k+1}>0, \ldots, w_{n}>0$, the system 47] is equivalent to:

$$
\left\{\begin{array}{l}
-y_{1, k+1} \cdot v_{1}-\ldots-y_{k, k+1} \cdot v_{k}+y_{k+1, k+1} \cdot w_{k+1}-\ldots-y_{k+1, n} \cdot w_{n}=\frac{P_{k+1}}{w_{k+1}} \\
\vdots \\
-y_{1, n} \cdot v_{1}-\ldots-y_{k, n} \cdot v_{k}-y_{k+1, n} \cdot w_{k+1}-\ldots+y_{n, n} \cdot w_{n}=\frac{P_{n}}{w_{n}}
\end{array}\right.
$$

and writing $(48)$ in matrix form, we obtain:

$$
-\Gamma_{k}^{t} \cdot V+\Lambda_{n-k} \cdot W=\Phi(W)
$$


where

$$
\Phi(W)=\Phi\left(\left(w_{k+1}, w_{k+2}, \ldots, w_{n}\right)^{t}\right)=\left(\frac{P_{k+1}}{w_{k+1}}, \frac{P_{k+2}}{w_{k+2}}, \ldots, \frac{P_{n}}{w_{n}}\right)^{t}
$$

here, it should be pointed out that $P_{k+i}$ are function of $w_{k+i}$, so $\Phi(W)=$ $\left(\frac{P_{k+1}\left(w_{k+1}\right)}{w_{k+1}}, \ldots, \frac{P_{n}\left(w_{n}\right)}{w_{n}}\right)^{t}$.

From expression (49), we deduce:

$$
-\Gamma_{k}^{t} \cdot \frac{\partial V}{\partial W}+\Lambda_{n-k}=\Phi^{\prime}(W)
$$

where

$$
\Phi^{\prime}(W)=\frac{\partial \Phi}{\partial W}=\left[\begin{array}{cccc}
\frac{1}{w_{k+1}} \frac{\partial P_{k+1}}{\partial w_{k+1}}-\frac{P_{k+1}}{w_{k+1}^{2}} & \frac{1}{w_{k+1}} \frac{\partial P_{k+1}}{\partial w_{k+2}} & \cdots & \frac{1}{w_{k+1}} \frac{\partial P_{k+1}}{\partial w_{n}} \\
\frac{1}{w_{k+2}} \frac{\partial P_{k+2}}{\partial w_{k+1}} & \frac{1}{w_{k+2}} \frac{\partial P_{k+2}}{\partial w_{k+2}}-\frac{P_{k+2}}{w_{k+2}^{2}} & \cdots & \frac{1}{w_{k+2}} \frac{\partial P_{k+2}}{\partial w_{n}} \\
\vdots & \vdots & \ddots & \vdots \\
\frac{1}{w_{n}} \frac{\partial P_{n}}{\partial w_{k+1}} & \frac{1}{w_{n}} \frac{\partial P_{n}}{\partial w_{k+2}} & \cdots & \frac{1}{w_{n}} \frac{\partial P_{n}}{\partial w_{n}}-\frac{P_{n}}{w_{n}^{2}}
\end{array}\right]
$$

and consequently:

$$
[W] \cdot \Phi^{\prime}(W)=\left[\begin{array}{cccc}
\frac{\partial P_{k+1}}{\partial w_{k+1}}-\frac{P_{k+1}}{w_{k+1}} & \frac{\partial P_{k+1}}{\partial w_{k+2}} & \ldots & \frac{\partial P_{k+1}}{\partial w_{n}} \\
\frac{\partial P_{k+2}}{\partial w_{k+1}} & \frac{\partial P_{k+2}}{\partial w_{k+2}}-\frac{P_{k+2}}{w_{k+2}} & \ldots & \frac{\partial P_{k+2}}{\partial w_{n}} \\
\vdots & \vdots & \ddots & \vdots \\
\frac{\partial P_{n}}{\partial w_{k+1}} & \frac{\partial P_{n}}{\partial w_{k+2}} & \cdots & \frac{\partial P_{n}}{\partial w_{n}}-\frac{P_{n}}{w_{n}}
\end{array}\right]
$$

and therefore:

$$
[W] \cdot \Phi^{\prime}(W)+[\Phi]=\left[\begin{array}{cccc}
\frac{\partial P_{k+1}}{\partial w_{k+1}} & \frac{\partial P_{k+1}}{\partial w_{k+2}} & \cdots & \frac{\partial P_{k+1}}{\partial w_{n}} \\
\frac{\partial P_{k+2}}{\partial w_{k+1}} & \frac{\partial P_{k+2}}{\partial w_{k+2}} & \cdots & \frac{\partial P_{k+2}}{\partial w_{n}} \\
\vdots & \vdots & \ddots & \vdots \\
\frac{\partial P_{n}}{\partial w_{k+1}} & \frac{\partial P_{n}}{\partial w_{k+2}} & \cdots & \frac{\partial P_{n}}{\partial w_{n}}
\end{array}\right]=\frac{\Pi}{\partial W}
$$

From equations (51) and (54), we deduce the expression (43), and if $\left[\mathbf{Y}_{k}-\Phi^{\prime}(V)\right]$ is invertible, then equation (44) is verified.

It is important to point out that $\Delta V \approx \frac{\partial V}{\partial W} \cdot \Delta W$, and $\Delta \Pi \approx \frac{\partial \Pi}{\partial W} \cdot \Delta W$, so that means that we can know how much vary some variables when the other change, because all of this information is in the respective Jacobian matrix.

Winpower/Alstom days - 5 \& 6 Ferbuary 2014. CONFIDENTIAL USE. NOT PUBLISHED 


\section{Application example. Four-terminal system.}

In order to show how our algorithm operates, a multi-terminal HVDC grid model shown in figure 2 is presented. In this model there are two wind producers nodes ( 1 and 2 ), two consumptions nodes (5 and 6 ). Also there are two interconnection nodes (3 and 4 ) which no power is injected or consumed. Table 1 lits the parameters values of this model.

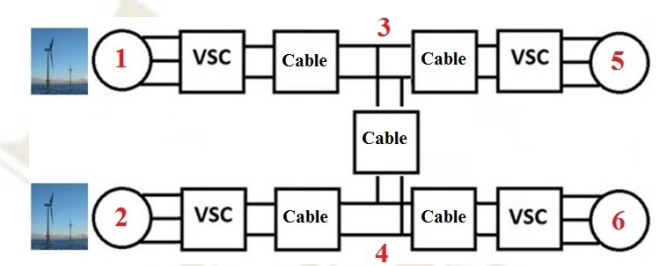

Fig. 2: General benchmark.

Tab. 1: Simulation values.

\begin{tabular}{|c|c|c|}
\hline$V_{\text {nom }}$ & Tab. 1: Simulation values. \\
\hline$P_{\text {nom-1 }}$ & Nominal power wind farm node 1 & $400 \mathrm{kV}$ \\
\hline$P_{\text {nom-2 }}$ & Nominal power wind farm node 2 & $300 \mathrm{MW}$ \\
\hline$R_{\text {cable }}$ & Cable resistance & $0.0121 \Omega / \mathrm{km}$ \\
\hline$L_{13}$ & Length line $1-3$ & $180 \mathrm{~km}$ \\
\hline$L_{24}$ & Length line $2-4$ & $200 \mathrm{~km}$ \\
\hline$L_{34}$ & Length line $3-4$ & $150 \mathrm{~km}$ \\
\hline$L_{35}$ & Length line $3-5$ & $100 \mathrm{~km}$ \\
\hline$L_{46}$ & Length line $4-6$ & $70 \mathrm{~km}$ \\
\hline
\end{tabular}

For these data, the admittance matrix of the network $\mathbf{Y}$ is:

$$
\mathbf{Y}=\left[\begin{array}{cccccc}
0.46 & 0 & -0.46 & 0 & 0 & 0 \\
0 & 0.41 & 0 & -0.41 & 0 & 0 \\
-0.46 & 0 & 1.84 & -0.55 & -0.83 & 0 \\
0 & -0.41 & -0.55 & 2.14 & 0 & -1.18 \\
0 & 0 & -0.83 & 0 & 0.83 & 0 \\
0 & 0 & 0 & -1.18 & 0 & 1.18
\end{array}\right]\left(\Omega^{-1}\right)
$$

As the nodes 5 and 6 are consumed nodes, we select these as nodes where the voltage is known. We will consider the power injected by the wind farms and the power in interconnection nodes as a input data for our algorithm. It could be noted that the power in interconnection nodes will be always zero in according with explained above. So according with the formulation explained in sections above, the variable $k$, the number of known power will be $k=4$, and the matrix $\mathbf{Y}_{k}=\mathbf{Y}_{4}$, and matrix $\boldsymbol{\Gamma}$ will be:

Winpower/Alstom days - 5 \& 6 Ferbuary 2014. CONFIDENTIAL USE. NOT PUBLISHED 


$$
\mathbf{Y}_{4}=\left[\begin{array}{cccc}
0.46 & 0 & -0.46 & 0 \\
0 & 0.41 & 0 & -0.41 \\
-0.46 & 0 & 1.84 & -0.55 \\
0 & -0.41 & -0.55 & 2.14
\end{array}\right] \quad \quad \boldsymbol{\Gamma}=\left[\begin{array}{cc}
0 & 0 \\
0 & 0 \\
0.83 & 0 \\
0 & 1.18
\end{array}\right]
$$

So, if for example, in a certain moment the wind farm 1 produces 200 MW and the wind farm 2 produces $100 \mathrm{MW}$, and we desire that the voltage at node 5 will be $399.5 \mathrm{kV}$, and the voltage at node will be $400 \mathrm{kV}$, the result of our algorithm, in steady state, will be shown in table 2 ;

Tab. 2: Power flow result

\begin{tabular}{cccccc}
\multicolumn{7}{c}{ Algorithm inputs } \\
\hline$P_{1}$ & $P_{2}$ & $P_{3}$ & $P_{4}$ & $u_{5}$ & $u_{6}$ \\
\hline $200 \mathrm{MW}$ & $100 \mathrm{MW}$ & $0 \mathrm{MW}$ & $0 \mathrm{MW}$ & $399.5 \mathrm{kV}$ & $400 \mathrm{kV}$ \\
& \multicolumn{7}{c}{ Results } \\
\hline$u_{1}$ & $u_{2}$ & $u_{3}$ & $u_{4}$ & $P_{5}$ & $P_{6}$ \\
$401.22 \mathrm{kV}$ & $400.79 \mathrm{kV}$ & $400.14 \mathrm{kV}$ & $400.19 \mathrm{kV}$ & $-210.3 \mathrm{MW}$ & $-88.63 \mathrm{MW}$
\end{tabular}

We observe as the voltages in nodes $1,2,3$ and 4 are close to the nominal value of the grid $(400 \mathrm{kV})$, and also as the consumed power in nodes 5 and 6 are within the limits. For these results the power losses in the grid due to cable resistances are 1.07 MW.

If we want to know how much varies the voltages and the power if one or some variables change, we use the Property 2. So if for example a change in the variables $\Delta u_{5}=0.5$ and $\Delta u_{6}=-0.3$, and using expressions $\Delta V \approx$ $\frac{\partial V}{\partial W} \cdot \Delta W$, and $\Delta \Pi \approx \frac{\partial \Pi}{\partial W} \cdot \Delta W:$

$$
\begin{gathered}
\Delta W=\left[\begin{array}{c}
0.5 \\
-0.3
\end{array}\right] \Rightarrow \frac{\delta V}{\delta W}=\left[\begin{array}{ll}
0.68 & 0.31 \\
0.22 & 0.78 \\
0.69 & 0.31 \\
0.22 & 0.78
\end{array}\right] \text { and } \frac{\partial \Pi}{\partial W}=\left[\begin{array}{cc}
102.89 & -103.02 \\
-103.16 & 103.28
\end{array}\right](57) \\
\Rightarrow[u]=\left[\begin{array}{c}
401.54 \\
400.67 \\
400.45 \\
400,08 \\
400.1 \\
399.7
\end{array}\right](k V) \text { and } P=\left[\begin{array}{c}
200 \\
100 \\
0 \\
0 \\
-127.94 \\
-171.19
\end{array}\right](M W)
\end{gathered}
$$

\section{Conclusions}

A new method to solve nonlinear equation systems in DC grids is exhibited. The main difference with old methods (power flows) is that there is no a 
unique node where the voltage is known (slack bus), and it will make safer the grid, because the voltages not only will depend on an unique node, but several nodes.

The results shown in this paper will be easily generalized for AC systems, where the reactive power appears, proceeding in analogous form with the same philosophy. new method to solve nonlinear equation systems in DC grids is exhibited. The main difference with old methods (power flow) is that there is no a unique node where the voltage is known (slack bus).

In addition, a detailed study of the variation of the power and voltages when these variables change is proved and tested, by means of the Jacobian matrix functions.

\section{Annex}

- If $x=\left(x_{1}, x_{2}, \ldots, x_{n}\right) \in \mathbb{R}^{n}$

$$
\begin{aligned}
\|x\|_{1} & =\left|x_{1}\right|+\left|x_{2}\right|+\ldots+\left|x_{n}\right| \\
\|x\|_{2} & =\sqrt{x_{1}^{2}+x_{2}^{2}+\ldots+x_{n}^{2}} \\
\|x\|_{\infty} & =\max \left\{\left|x_{1}\right|+\left|x_{2}\right|+\ldots+\left|x_{n}\right|\right\} \\
\|x\|_{\infty} & \leq\|x\|_{2} \leq\|x\|_{1}
\end{aligned}
$$

- If $a \in \mathbb{R}^{n}$ and $0<r \in \mathbb{R}$ (with $\mathrm{p}=1,2$ or $\infty$ )

$$
\begin{aligned}
& B_{p}(a, r)=\left\{x \in \mathbb{R}^{n}:\|x-a\|_{p}<r\right\} \\
& \bar{B}_{p}(a, r)=\left\{x \in \mathbb{R}^{n}:\|x-a\|_{p} \leq r\right\} \\
& B_{1}(a, r) \subset B_{2}(a, r) \subset B_{\infty}(a, r) \\
& \bar{B}_{1}(a, r) \subset \bar{B}_{2}(a, r) \subset \bar{B}_{\infty}(a, r)
\end{aligned}
$$

- If $A \in \mathbb{R}^{m, n}$ (with $\mathrm{p}=1,2$ or $\infty$ )

$$
\begin{aligned}
& \|A\|_{p}=\max _{x \neq 0} \frac{\|A x\|_{p}}{\|x\|_{p}}=\max _{\|x\|_{p}=1}\|A x\|_{p} \\
& \|A \cdot B\|_{p} \leq\|A\|_{p} \cdot\|B\|_{p} \text { where } B \in \mathbb{R}^{n, l}
\end{aligned}
$$

$\|A\|_{1}=\max \left\{\left\|A_{1}\right\|_{1},\left\|A_{2}\right\|_{1}, \ldots,\left\|A_{n}\right\|_{1}\right\}$ (the maximum of norm one of the columns of $\mathrm{A}$ ).

$\|A\|_{\infty}=\max \left\{\left\|A^{1}\right\|_{1},\left\|A^{2}\right\|_{1}, \ldots,\left\|A^{n}\right\|_{1}\right\}$ (the maximum of norm one of the rows of $\mathrm{A}$ ).

- $\Psi\left(\left(v_{1}, v_{2}, \ldots, v_{k}\right)^{t}\right)=\left(\frac{P_{1}}{v_{1}}, \frac{P_{2}}{v_{2}}, \ldots, \frac{P_{k}}{v_{k}}\right)^{t}=\left(i_{1}, i_{2}, \ldots, i_{k}\right)^{t}$ 


$$
\Psi^{\prime}(V)=\left[\begin{array}{cccc}
-\frac{P_{1}}{v_{1}^{2}} & 0 & \ldots & 0 \\
0 & -\frac{P_{2}}{v_{2}^{2}} & \ldots & 0 \\
\vdots & \vdots & \ddots & \vdots \\
0 & 0 & \ldots & -\frac{P_{k}}{v_{k}^{2}}
\end{array}\right], \mathbf{Y}_{k}-\Psi^{\prime}(V)=\left[\begin{array}{cccc}
y_{1,1}+\frac{P_{1}}{v_{1}^{2}} & -y_{1,2} & \ldots & -y_{1, k} \\
-y_{1,2} & y_{2,2}+\frac{P_{2}}{v_{2}^{2}} & \ldots & -y_{2, k} \\
\vdots & \vdots & \ddots & \vdots \\
-y_{1, k} & -y_{2, k} & \ldots & y_{k, k}+\frac{P_{k}}{v_{k}^{2}}
\end{array}\right]
$$

- The equation (41) could be written as:

$$
\left[\begin{array}{cccc}
y_{1,1}+\frac{P_{1}}{v_{1}^{2}} & -y_{1,2} & \ldots & -y_{1, k} \\
-y_{1,2} & y_{2,2}+\frac{P_{2}}{v_{2}^{2}} & \ldots & -y_{2, k} \\
\vdots & \vdots & \ddots & \vdots \\
-y_{1, k} & -y_{2, k} & \ldots & y_{k, k}+\frac{P_{k}}{v_{k}^{2}}
\end{array}\right] \cdot\left[\begin{array}{ccc}
\frac{\partial v_{1}}{\partial w_{k+1}} & \ldots & \frac{\partial v_{1}}{\partial w_{n}} \\
\frac{\partial v_{2}}{\partial w_{k+1}} & \ldots & \frac{\partial v_{2}}{\partial w_{n}} \\
\vdots & \ddots & \ldots \\
\frac{\partial v_{n}}{\partial w_{k+1}} & \ldots & \frac{\partial v_{n}}{\partial w_{n}}
\end{array}\right]=\left[\begin{array}{ccc}
y_{1, k+1} & \ldots & y_{1, n} \\
y_{2, k+1} & \ldots & y_{2, n} \\
\vdots & \ddots & \ldots \\
y_{k, k+1} & \ldots & y_{k, n}
\end{array}\right]
$$

- $\Phi\left(\left(w-k+1, w_{k+2}, \ldots, w_{n}\right)^{t}\right)=\left(\frac{P_{k+1}}{w_{k+1}}, \frac{P_{k+2}}{w_{k+2}}, \ldots, \frac{P_{n}}{w_{n}}\right)^{t}=\left(i_{k+1}, i_{k+2}, \ldots, i_{n}\right)^{t}$, where:

$$
[\Phi]=\left[\begin{array}{cccc}
-\frac{P_{k+1}}{w_{1}} & 0 & \ldots & 0 \\
0 & -\frac{P_{k+2}}{w_{2}} & \ldots & 0 \\
\vdots & \vdots & \ddots & \vdots \\
0 & 0 & \ldots & -\frac{P_{n}}{w_{n}}
\end{array}\right], \quad[W]=\left[\begin{array}{cccc}
w_{k+1} & 0 & \ldots & 0 \\
0 & w_{k+2} & \ldots & 0 \\
\vdots & \vdots & \ddots & \vdots \\
0 & 0 & \ldots & w_{n}
\end{array}\right]
$$

- The equation (43) could be written as:

$$
\left[\begin{array}{ccc}
\frac{\partial P_{k+1}}{\partial w_{k+1}} & \ldots & \frac{\partial P_{k+1}}{\partial w_{n}} \\
\vdots & \ddots & \vdots \\
\frac{\partial P_{n}}{\partial w_{k+1}} & \cdots & \frac{\partial P_{n}}{\partial w_{n}}
\end{array}\right]=\left[\begin{array}{ccc}
\frac{P_{k+1}}{w_{k+1}} & \ldots & 0 \\
\vdots & \ddots & \vdots \\
0 & \ldots & \frac{P_{n}}{w_{n}}
\end{array}\right]+\left[\begin{array}{ccc}
w_{k+1} & \ldots & 0 \\
\vdots & \ddots & \vdots \\
0 & \ldots & w_{n}
\end{array}\right] \cdot\left[\Gamma_{n-k}-\Lambda_{k}^{t} \cdot \frac{\partial V}{\partial W}\right]
$$

where:

$$
\left[\Gamma_{n-k}-\Lambda_{k}^{t} \cdot \frac{\partial V}{\partial W}\right]=\left[\begin{array}{ccc}
y_{k+1, k+1} & \ldots & -y_{k+1, n} \\
\vdots & \ddots & \vdots \\
-y_{k+1, n} & \ldots & y_{n, n}
\end{array}\right]-\left[\begin{array}{ccc}
y_{1, k+1} & \ldots & y_{k, k+1} \\
\vdots & \ddots & \vdots \\
y_{1, n} & \ldots & y_{k, n}
\end{array}\right] \cdot\left[\begin{array}{ccc}
\frac{\partial v_{1}}{\partial w_{k+1}} & \ldots & \frac{\partial v_{1}}{\partial w_{n}} \\
\vdots & \ddots & \vdots \\
\frac{\partial v_{k}}{\partial w_{k+1}} & \ldots & \frac{\partial v_{k}}{\partial w_{n}}
\end{array}\right]
$$




\section{References}

[1] J. Arrillaga, Y. Liu, and N. Watson, Flexible Power Transmission. The HVDC Options. John Wiley \& Sons Ltd, 2007.

[2] G. Asplund, "Ultra high voltaje trasmission," ABB review, February 2007.

[3] J. Kreuse, "The future is now," ABB review, March 2008.

[4] D. Ravemark and B. Normark, "Light and invisible," ABB review, March 2005.

[5] A. Sannino, P. Sandeberg, L. Stendius, and R. Görner, "Enabling the power of wind," Tech. Rep.

[6] R. Rudervall, J. Charpentier, and R. Sharma, "High voltage direct current (hvdc) transmission systems. technology review paper," Tech. Rep.

[7] P. Kundur, Power systems stability and control, 1st ed. Mc Graw Hill, 1993.

[8] M. Jiménez-Carrizosa, F. Dorado-Navas, G. Damm, and F. LamnabhiLagarrigue, "Optimal power flow in multi-terminal hvdc grids with offshore wind farms and storage devices." 